\title{
Photometric observations of Rosetta target asteroid 2867 Steins
}

\author{
P. R. Weissman ${ }^{1}$, S. C. Lowry ${ }^{2}$, and Y.-J. Choi ${ }^{1}$ \\ 1 Science Division, Jet Propulsion Laboratory, 4800 Oak Grove Drive, MS 183-301, Pasadena, CA 91109, USA \\ e-mail: [paul.r.weissman; young-jun.choi]@jpl.nasa.gov \\ 2 School of Mathematics and Physics, Queen's University Belfast, Belfast, BT7 1NN, UK \\ e-mail: s.c.lowry@qub.ac.uk
}

Received 16 September 2006 / Accepted 30 January 2007

\begin{abstract}
Asteroid 2867 Steins is one of two flyby targets of ESA's International Rosetta Mission, launched in March, 2004. We obtained CCD observations of Steins on April 14-16, 2004 at Table Mountain Observatory, California, in order to characterize the asteroid physically, information that is crucial for planning the Steins flyby. This study includes the first detailed analysis of the physical properties of Steins from time-series $R$-filter data along with $V$ - and $I$-filter photometric measurements. We found a mean $R$-filter absolute magnitude of $12.60 \pm 0.02$ (for $G=0.15$ ), corresponding to a mean radius of $3.57 \pm 0.03 \mathrm{~km}$ assuming an S-type reflectance of 0.20 , or $2.24 \pm 0.02 \mathrm{~km}$ assuming an E-type reflectance of 0.40 (and $G=0.40$ ). The observed brightness range of $0.29 \pm 0.04 \mathrm{mag}$ suggests a lower limit on the axial ratio, $a / b$, of 1.30 . We determined a synodic rotation period of $6.048 \pm 0.007 \mathrm{~h}$, assuming a doublepeaked lightcurve. We fitted the available $R$-filter photometry over the phase angle range of 11.08-17.07 degrees and found best-fit phase function parameters of $G=0.46_{-0.20}^{+0.32}$, and $H=12.92_{-0.17}^{+0.22}$. Derived colour indices for the asteroid are $(V-R)=0.58 \pm 0.03$, and $(R-I)=0.44 \pm 0.03$. These values are consistent with, though slightly redder than Hicks et al. (IAUC 8315). Barucci et al. (2005) identified Steins as an E-type based on visual and near-infrared spectra, but if that is correct, then it is an unusually red E-type asteroid.
\end{abstract}

Key words. minors planets, asteroids

\section{Introduction}

Asteroid 2867 Steins is one of two flyby targets of the European Space Agency's International Rosetta Mission, launched on March 2, 2004. Rosetta will rendezvous with periodic comet 67P/Churyumov-Gerasimenko in 2014. En route to the comet, Rosetta will fly by two main belt asteroids, 2867 Steins on September 5, 2008 and 21 Lutetia on July 10, 2010. As the targets were changed due to the Rosetta launch delay from 2003 to 2004, the new target comet and flyby asteroids have little observational data available (with the exception of Lutetia). Thus, new studies like this one are needed to build up a detailed picture of the physical and surface characteristics of asteroid Steins.

Asteroid 2867 Steins is located in the inner main belt at a semi-major axis of $2.363 \mathrm{AU}$. Orbital elements for the asteroid are given in Table 1. Its absolute $R$-band magnitude of 12.67 from unpublished measurements (Hicks et al. IAUC 8315) corresponds to a mean diameter of $6.9 \mathrm{~km}$ if it is a typical S-type with albedo $=0.20$ or $4.9 \mathrm{~km}$ for an E-type object with albedo $=$ 0.40 . Hicks et al. found $B V R I$ colours consistent with an S-type taxonomic classification, though they could not rule out a Dtype object. However, Barucci et al. (2005) classified 2867 Steins as an E-type asteroid based on visual and near-infrared spectra taken in January and May, 2004. E-type asteroids are similar to enstatite achondrite meteorites, one of the more thermally processed classes.

Polarization curves at wavelengths of $R$ and $V$ passbands were obtained by Fornasier et al. (2006) from observations with ESO's VLT telescope, and suggest an albedo of $0.45 \pm 0.10$. Unfortunately, with this method there is a large scatter in the albedo/polarimetric-slope relation that will inevitably lead to
Table 1. Orbital elements: 2867 Steins.

\begin{tabular}{ll}
\hline \hline Element & Value $^{a}$ \\
\hline semimajor axis & $2.3633 \mathrm{AU}$ \\
eccentricity & 0.145478 \\
inclination & 9.9456 degrees \\
argument of perihelion & 250.4844 degrees \\
longitude of ascending node & 55.5399 degrees \\
time of perihelion & 2001 Nov. 04.21279 \\
\hline
\end{tabular}

${ }^{a}$ Epoch: 2005-Jan.-30.

a large uncertainty in the implied albedo. Preliminary analysis of observations of Steins with the Spitzer Space Telescope, combined with visual data, suggest a somewhat lower albedo of 0.30-0.40 (Lamy et al. 2006).

We observed 2867 Steins in April, 2004 to characterize the asteroid physically. Knowledge of the asteroid's size, shape, rotation period, and spectral behaviour are crucial in planning the science observations of Rosetta. In addition, lightcurves obtained at multiple apparitions can be used to derive a full threedimensional shape model and rotation pole orientation, again very important for planning of the Steins flyby. This was done with great success by Kaasalainen et al. (2003) for asteroid 25143 Itokawa, target of the Hayabusa sample return mission. Comparison of spacecraft observations with results derived from telescopic observations also provides critical ground truth for remote observing and data analysis techniques.

This paper is organized as follows. Section 2 describes the observational configuration and strategy employed for this programme. Section 3 describes the analysis techniques that were 
Table 2. Observing geometry (at 4 h UT).

\begin{tabular}{lllc}
\hline \hline Date & $r(\mathrm{AU})$ & $\Delta(\mathrm{AU})$ & Phase angle (deg.) \\
\hline 14 April 2004 & 2.5601 & 1.7731 & 16.78 \\
15 April 2004 & 2.5588 & 1.7809 & 17.07 \\
16 April 2004 & 2.5576 & 1.7890 & 17.35 \\
\hline
\end{tabular}

applied to the data, and the results on the bulk physical and surface properties are also presented. A summary of the results and main conclusions is given is the final section.

\section{Observations of 2867 Steins}

Asteroid 2867 Steins was observed with the $0.6 \mathrm{~m}$, RitcheyChretien telescope at the Table Mountain Observatory (TMO), near Wrightwood, California on April 14-16, 2004 (UT). The observing geometry of the asteroid on each date is listed in Table 2. Images were obtained with the facility CCD camera, using a Photometrics $1024 \times 1024$ pixel thinned and backilluminated CCD, mounted at the Cassegrain focus of the f/16 telescope. The pixel scale was $0.52^{\prime \prime} /$ pixel and the total field-of-view was $8.9^{\prime} \times 8.9^{\prime}$. For all images the telescope was tracked at the asteroid's predicted rate of motion based on an ephemeris from JPL's Horizons system (Giorgini \& Yeomans 1999).

The asteroid was located slightly past opposition and at a northern declination of $+20^{\circ}$, allowing observations below two air masses for about six hours per night. Observations were conducted using the Johnson/Kron-Cousins VRI-filter set. A total of $38 R$-filter images of the asteroid field were obtained on night 1 , 36 on night 2 , and 39 on night 3 . Typical exposure times were $360-600$ s. Additionally, on night 2, one $V$-filter and one $I$-filter image each were obtained as part of an $R-V-R-I-R$ sequence. Each night's observations included twilight sky frames in the $R$-filter (plus the $V$ and $I$ filters on night 2), bias (zero exposure) frames, and Landolt fields (Landolt 1992) imaged over a range of air masses. Observing conditions on nights 1 and 3 were photometric while night 2 suffered from some high cirrus. Because of this, calibration frames of the Steins star fields for night 2 were obtained on a later run at TMO under photometric conditions. The Landolt fields used were PG1047+003, PG1323-086, and PG1528+062 (Landolt 1992).

\section{Data reduction and analysis}

All images were bias-subtracted and flat-fielded, and other instrumental artifacts, such as cosmic rays and bad rows/columns were removed in the standard manner. Standard aperture photometry was applied to the images using an aperture $\sim 3$ times the maximum measured seeing each night. Image processing was performed using the Image Reduction and Analysis Facility (IRAF) (Tody 1986, 1993).

\subsection{Shape, size, and surface colour}

A relative asteroid lightcurve was extracted from the imaging for each night by comparing the asteroid's $R$-filter brightness with that of non-varying field stars in the same images. The observed rotational lightcurve was asymmetric, and the observed full brightness range was $0.29 \pm 0.04 \mathrm{mag}$. This implies an axial ratio, $a / b$, of $\geq 1.30 \pm 0.04$, using $a / b \geq 10^{0.4 \Delta m}$, where $a$ and $b$ are the semi-axes of the asteroid and $\Delta m$ is the range of observed magnitudes. This simple model assumes a bi-axial ellipsoid shape and uniform surface albedo, and ignores phase effects. Zappalà et al. (1990) analysed the relationship between lightcurve amplitudes and phase angles for a subset of known asteroid classes. They showed that the lightcurve amplitude increases with phase angle, and that the degree of change is dependent on the taxonomic class of the asteriod. More specifically,

$\Delta m\left(0^{\circ}\right)=\frac{\Delta m(\alpha)}{1+\kappa \alpha}$

where $\Delta m\left(0^{\circ}\right)$ and $\Delta m(\alpha)$ are the lightcurve amplitudes at $0^{\circ}$ and $\alpha^{\circ}$. The constant $\kappa\left[\mathrm{deg}^{-1}\right]$ has values of $0.030,0.015,0.014$, and 0.013 for $\mathrm{S}, \mathrm{M}, \mathrm{R}$, and $\mathrm{C}$ asteroid taxonomic types, respectively. Taking the $\kappa$ value for S-types and applying Eq. (1) gives $\Delta m\left(0^{\circ}\right)=0.188$ and a corrected axial ratio of 1.19 . Unfortunately, no $\kappa$ value is available for E-type asteroids, therefore we use the S-type value based on the very red colour indices of 2867 Steins (see below).

The calibrated apparent magnitudes from our Table Mountain run on April 14-16, 2005 are listed in Table 3. The mean calibrated $R$-filter apparent magnitude, or the apparent magnitude at lightcurve mid-point is $16.80 \pm 0.02$. If we assume an S-type phase-slope parameter G of 0.15 in the standard HG system of Bowell et al. (1989), as used by Hicks et al. (IAUC 8315), we obtain a mean absolute $R$-filter magnitude of $12.60 \pm 0.02$. This is brighter by 0.07 mag than the Hicks et al. measurement, but consistent at the $2 \sigma$ level. Alternatively, if we assume a typical $\mathrm{G}$ value of 0.40 for an E-type asteroid, we obtain a mean absolute $R$-filter magnitude of $12.86 \pm 0.02$.

Assuming a typical S-type albedo of 0.20 (and S-type $\mathrm{G}$ value), our mean apparent $R$-filter magnitude implies a mean effective radius of $3.57 \pm 0.03 \mathrm{~km}$ or semi-axes of $3.42 \pm 0.03$ and $4.06 \pm 0.03 \mathrm{~km}$ if the phase-angle-corrected lightcurve magnitude range is considered. Assuming a typical E-type albedo of 0.40 (and E-type $\mathrm{G}$ value of 0.40 ), our mean apparent magnitude implies a smaller mean effective radius of $2.24 \pm 0.02 \mathrm{~km}$ with semi-axes of $2.14 \pm 0.02$ and $2.54 \pm 0.02 \mathrm{~km}$, again using the corrected axial ratio of 1.19 . We use the size-magnitude relationship of Russell (1916).

To remove the confounding effects on the derived colour indices due to possible projected area variation with rotation, we compared the $V$ and $I$ apparent magnitudes with interpolated $R$-filter measurements from Table 3 . We took the average of the two bracketing $R$-filter data points for each of the $V$ and $I$ measurements, and measured the difference to get the $(V-R)$ and $(R-I)$ colour indices. Our quoted $(V-I)$ is the summation of these two colour indices and not the difference between the actual $V$ and $I$ apparent magnitudes.

The interpolated colour indices are given in Table 4, along with the corresponding $R$ magnitude, and the values found by Hicks et al. (IAUC 8315). Our values are somewhat redder than Hicks et al. but consistent at the $1.2 \sigma$ level. In Fig. 1 we compare these colours with similar colour indices for other asteroids, derived from ECAS data (1985) using the transformation method discussed in Dandy et al. (2003). Based on our colours we find that the visible colours for this asteroid are much redder than typical E-type asteroids. As noted above, Barucci et al. (2005) report an E-type classification for Steins based on visual and near-infrared spectra, in particular the presence of a $0.49 \mu \mathrm{m}$ absorption band. New preliminary colour data from Weissman et al. (personal communication), based on observations from Cerro Tololo in August 2005, give results similar to both Hicks et al. and our results presented here. Thus, all available visible colours of 2867 Steins agree. We compare our results with those 
Table 3. Apparent $R$-filter magnitudes.

\begin{tabular}{|c|c|c|c|c|c|}
\hline Date $^{\dagger}$ & $m_{R} \pm \sigma$ & Date $^{\dagger}$ & $m_{R} \pm \sigma$ & Date $^{\dagger}$ & $m_{R} \pm \sigma$ \\
\hline 09.62304 & $16.763 \pm 0.032$ & 10.62904 & $16.811 \pm 0.020$ & 11.62060 & $16.808 \pm 0.024$ \\
\hline 09.62857 & $16.771 \pm 0.030$ & 10.63420 & $16.771 \pm 0.018$ & 11.62598 & $16.790 \pm 0.023$ \\
\hline 09.63371 & $16.787 \pm 0.030$ & 10.63939 & $16.801 \pm 0.018$ & 11.63113 & $16.761 \pm 0.018$ \\
\hline 09.63990 & $16.858 \pm 0.028$ & 10.65213 & $16.901 \pm 0.019$ & 11.63612 & $16.761 \pm 0.018$ \\
\hline 09.64901 & $16.876 \pm 0.028$ & 10.65789 & $16.891 \pm 0.018$ & 11.64205 & $16.758 \pm 0.019$ \\
\hline 09.65426 & $16.875 \pm 0.028$ & 10.66877 & $16.941 \pm 0.018$ & 11.64699 & $16.796 \pm 0.019$ \\
\hline 09.68235 & $16.731 \pm 0.027$ & 10.67967 & $16.881 \pm 0.019$ & 11.65202 & $16.847 \pm 0.018$ \\
\hline 09.68752 & $16.710 \pm 0.029$ & 10.74053 & $16.741 \pm 0.018$ & 11.65723 & $16.892 \pm 0.018$ \\
\hline 09.69274 & $16.698 \pm 0.029$ & 10.74587 & $16.751 \pm 0.018$ & 11.66232 & $16.926 \pm 0.017$ \\
\hline 09.69778 & $16.708 \pm 0.029$ & 10.75133 & $16.761 \pm 0.018$ & 11.66766 & $16.974 \pm 0.018$ \\
\hline 09.70309 & $16.722 \pm 0.030$ & 10.75642 & $16.781 \pm 0.018$ & 11.67288 & $16.993 \pm 0.018$ \\
\hline 09.70796 & $16.698 \pm 0.030$ & 10.76155 & $16.751 \pm 0.018$ & 11.69066 & $16.874 \pm 0.018$ \\
\hline 09.71323 & $16.671 \pm 0.029$ & 10.76674 & $16.791 \pm 0.018$ & 11.69583 & $16.858 \pm 0.017$ \\
\hline 09.71812 & $16.681 \pm 0.029$ & 10.77238 & $16.821 \pm 0.019$ & 11.70094 & $16.772 \pm 0.018$ \\
\hline 09.72344 & $16.652 \pm 0.030$ & 10.77748 & $16.811 \pm 0.018$ & 11.70624 & $16.772 \pm 0.017$ \\
\hline 09.72992 & $16.671 \pm 0.028$ & 10.78270 & $16.841 \pm 0.019$ & 11.71160 & $16.753 \pm 0.017$ \\
\hline 09.73527 & $16.706 \pm 0.029$ & 10.78789 & $16.861 \pm 0.019$ & 11.71813 & $16.755 \pm 0.017$ \\
\hline 09.75697 & $16.803 \pm 0.029$ & 10.79314 & $16.871 \pm 0.019$ & 11.72325 & $16.751 \pm 0.017$ \\
\hline 09.76257 & $16.866 \pm 0.028$ & 10.79837 & $16.911 \pm 0.019$ & 11.72834 & $16.737 \pm 0.017$ \\
\hline 09.76782 & $16.837 \pm 0.028$ & 10.81798 & $16.871 \pm 0.020$ & 11.73433 & $16.758 \pm 0.017$ \\
\hline 09.77325 & $16.806 \pm 0.030$ & 10.82328 & $16.811 \pm 0.019$ & 11.74071 & $16.776 \pm 0.017$ \\
\hline 09.77860 & $16.829 \pm 0.030$ & 10.82836 & $16.801 \pm 0.018$ & 11.74583 & $16.767 \pm 0.017$ \\
\hline 09.78385 & $16.873 \pm 0.029$ & 10.83333 & $16.791 \pm 0.019$ & 11.75075 & $16.753 \pm 0.017$ \\
\hline 09.78904 & $16.872 \pm 0.029$ & 10.83837 & $16.761 \pm 0.019$ & 11.77404 & $16.860 \pm 0.018$ \\
\hline 09.79431 & $16.883 \pm 0.029$ & 10.84334 & $16.711 \pm 0.018$ & 11.77997 & $16.889 \pm 0.017$ \\
\hline 09.79971 & $16.853 \pm 0.029$ & 10.84851 & $16.751 \pm 0.019$ & 11.78600 & $16.840 \pm 0.018$ \\
\hline 09.80495 & $16.839 \pm 0.028$ & 10.85445 & $16.731 \pm 0.019$ & 11.79130 & $16.841 \pm 0.017$ \\
\hline 09.81022 & $16.776 \pm 0.030$ & 10.85966 & $16.711 \pm 0.020$ & 11.79699 & $16.846 \pm 0.018$ \\
\hline 09.83800 & $16.764 \pm 0.029$ & 10.86466 & $16.741 \pm 0.020$ & 11.80215 & $16.869 \pm 0.018$ \\
\hline 09.84326 & $16.697 \pm 0.030$ & 10.86982 & $16.751 \pm 0.019$ & 11.80769 & $16.845 \pm 0.019$ \\
\hline 09.84917 & $16.730 \pm 0.031$ & 10.88739 & $16.821 \pm 0.020$ & 11.81269 & $16.890 \pm 0.017$ \\
\hline 09.85447 & $16.665 \pm 0.030$ & 10.89260 & $16.771 \pm 0.021$ & 11.81770 & $16.849 \pm 0.018$ \\
\hline 09.85960 & $16.699 \pm 0.031$ & 10.89749 & $16.861 \pm 0.019$ & 11.82274 & $16.854 \pm 0.018$ \\
\hline 09.86487 & $16.699 \pm 0.030$ & 10.90264 & $16.831 \pm 0.020$ & 11.82770 & $16.824 \pm 0.019$ \\
\hline 09.87026 & $16.732 \pm 0.032$ & 10.90776 & $16.901 \pm 0.021$ & 11.83280 & $16.807 \pm 0.018$ \\
\hline 09.87556 & $16.747 \pm 0.032$ & 10.91278 & $16.881 \pm 0.020$ & 11.85688 & $16.766 \pm 0.018$ \\
\hline 09.88091 & $16.791 \pm 0.034$ & - & - & 11.88991 & $16.800 \pm 0.018$ \\
\hline 09.88637 & $16.827 \pm 0.037$ & - & - & 11.89593 & $16.853 \pm 0.020$ \\
\hline- & - & - & - & 11.90191 & $16.845 \pm 0.020$ \\
\hline
\end{tabular}

$\dagger$ - Light-time corrected mid-exposure-JD minus 2453100.

Table 4. BVRI colours for Asteroid 2867 Steins.

\begin{tabular}{cccc}
\hline \hline Colour & This work & $\bar{R}^{\dagger}$ & Hicks et al. (IAUC 8315) \\
\hline$V-R$ & $0.58 \pm 0.03$ & $16.91 \pm 0.02$ & $0.51 \pm 0.03$ \\
$R-I$ & $0.44 \pm 0.03$ & $16.92 \pm 0.02$ & $0.44 \pm 0.03$ \\
$B-R$ & - & & $1.311 \pm 0.03$ \\
\hline
\end{tabular}

$\dagger$ - Corresponding average apparent $R$ magnitude (see text).

of Hicks et al. (2004) and the Barucci et al. (2005) spectrum in Fig. 2. All three data sets of Steins agree on its strong red colour. If Steins is an E-type asteroid, then it is unusually red.

The differences in our colours and those by Hicks et al. (2004) may be indicative of surface inhomogeneities, resulting in areas of differing spectral slopes, perhaps from shifting of surface regolith. Such a mechanism could explain the fascinating surface characteristics of asteroid Itokawa as observed by the Hayabusa asteroid-rendezvous spacecraft (Fujiwara et al. 2006; Abe et al. 2006). This issue could be addressed by obtaining rotationally resolved spectra of 2867 Steins. Otherwise, it must await the examination of Steins by the Rosetta spacecraft in 2008 .

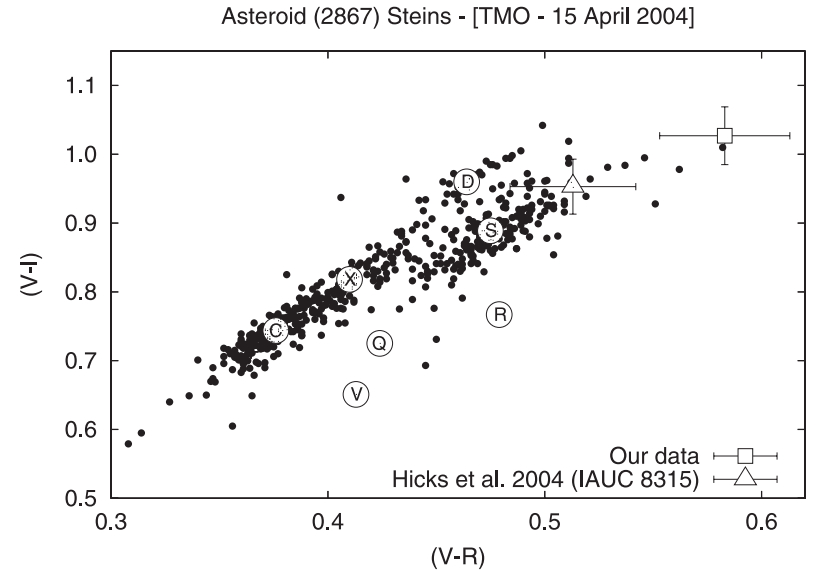

Fig. 1. Comparison of our colours with Hicks et al. (IAUC 8315), and ECAS data from Zellner et al. (1985). The positions of the main Tholen classes are marked (Tholen 1984). Our colour data is consistent with Hicks et al. at the $1.2 \sigma$ level, but are much redder than typical E-type (i.e. X-type) bodies. Barucci et al. (2005) assign an E-type classification to Steins due to the presence of a $0.49 \mu \mathrm{m}$ absorption feature in their spectrum. 


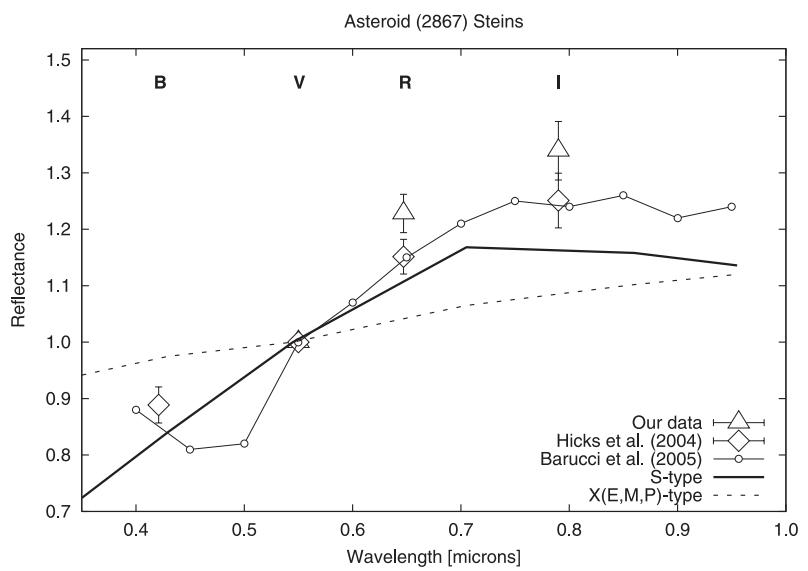

Fig. 2. Here we compare the available visual broad-band photometry and spectroscopy for Steins. We include the reflectance spectrum from Barucci et al. (2005), which has been sampled at $0.05 \mu \mathrm{m}$ intervals, and normalized at $0.55 \mu \mathrm{m}$. The broadband colours have been converted to reflectances and normalized at $0.55 \mu \mathrm{m}$ (i.e. at $V$-filter wavelengths). Our VRI data is included, along with $B V R I$ photometry from Hicks et al. (IAUC 8315). One can see that the Barucci et al. spectrum agrees very well with the Hicks et al. data set. Our data is slightly redder than the rest, but still in agreement within the photometry uncertainties and the noise level of the spectrum. We have also included mean reflectance spectra for the Tholen X-and S-types. The photometry and spectroscopy all show that the spectrum is much redder than a typical E-type, though there are a few E-type outliers that approach the redness of the Steins spectrum.

\subsection{Photometric phase function}

We used the available $R$-filter photometry to assess the phaseangle variation of the asteroid's brightness. Our data are combined with the Hicks et al. data (IAUC 8315) to perform a fit of the photometric phase function in terms of the HG formalism. The Hicks et al. data and our data were taken when Steins was at average phase angles of 11.07 and 17.07 degrees, respectively. For the fit we use Hicks et al's. mean apparent magnitude of $16.53 \pm 0.02$ and convert to "reduced" magnitude $R(1,1, \alpha)$, i.e. the apparent magnitude has been scaled to unit heliocentric and geocentric distances. The reduced magnitude is $13.36 \pm 0.02$. We do the same for our mean $R$-filter apparent magnitude of $16.80 \pm 0.02$, and the resulting reduced magnitude is $13.51 \pm 0.02$.

The best fit photometric values are $G=0.46$ and $H=12.92$. When the $1 \sigma$ photometric uncertainties in the mean apparent magnitudes are considered we find that $\mathrm{G}$ values of 0.26 and 0.78 fit the data just as well. The corresponding $\mathrm{H}$ values are 12.75 and 13.14 , respectively, and the fits are shown graphically in Fig. 3. The accepted phase function parameters and $1 \sigma$ uncertainties are therefore $G=0.46_{-0.20}^{+0.32}$, and $H=12.92_{-0.17}^{+0.22}$. Although there is no phase coverage within the opposition-surge region at small phase angles we still prefer to fit for $G$ rather than simply applying a linear phase law that is inappropriate for asteroidal bodies.

A shallow slope parameter of $G=0.46$ is more associated with E-type asteroids than S-type. Because of the large error bar we cannot put too much weight on our derived slope parameter as a means of distinguishing whether or not the asteroid is either type. At the very least, this would require refinement of our phase-slope measurement through opposition surge coverage along with observations at large phase angles. Nevertheless, this result will aid in better assessment of the brightness of Steins for future observational planning, which needs to take place at a wide range of observational geometries, allowing for the full 3D

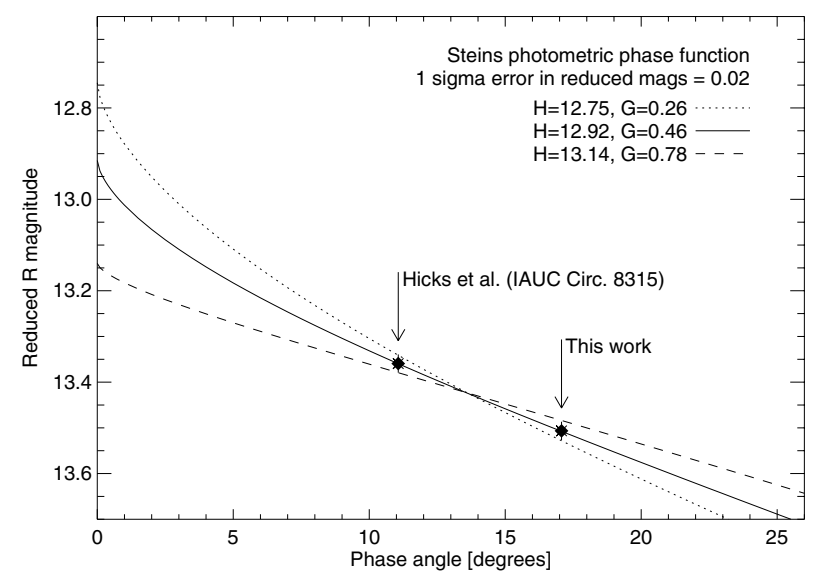

Fig. 3. We use the available $R$-filter photometry for a preliminary assessment of the phase-angle variation of the asteroid's brightness in terms of the HG formalism. The best-fit phase function parameters and associated $1 \sigma$ uncertainties are $G=0.46_{-0.20}^{+0.32}$, and $H=12.92_{-0.17}^{+0.22}$. Oppositionsurge coverage would be useful along with observations at large phase angles in order to refine this measurement.

shape and orientational modelling to take place. Taken together, these data will also allow a full Hapke-type analysis to be performed (e.g. Lederer et al. 2005), providing detailed information on Steins' surface regolith.

For completeness, we compute the corresponding effective radius using this fitted $\mathrm{H}$ value and for assumed typical geometric albedos of both S-type and E-type asteroids. For a typical S-type albedo of 0.20 , the absolute magnitude corresponds to an effective radius of $3.08_{-0.30}^{+0.25} \mathrm{~km}$, and for an E-type albedo of 0.40 , the absolute magnitude corresponds to an effective radius of $2.18_{-0.21}^{+0.18} \mathrm{~km}$.

\subsection{Rotational properties}

We applied the method of Harris et al. (1989) to determine the rotation period. This method involves fitting an nth-order Fourier series to the relative magnitudes, which is then repeated for a wide range of periods until the fit residuals are minimized. The chosen range of periods depends on initial inspection of the lightcurves. When fitting model lightcurves to the data, we start with low order fits to get a feel for where the prominent periodicities reside. We then increase the order to refine the dominant periodicity and its associated uncertainty, and also the quality of the fit. We stop increasing the order once the quality of the fit no longer improves. It was clear from our initial inspection of the data that the asteroid was rotating in $\sim 6 \mathrm{~h}$, assuming a doublepeaked lightcurve. We created a periodogram over the reasonable range of $0-15 \mathrm{~h}$, which is shown in the upper panel of Fig. 4. This periodogram results from 2 nd order fits and shows the location of two dominant periodicities. As we know the object has a full rotation period of just over $6 \mathrm{~h}$ (assuming a double-peaked lightcurve), we adopt the feature at $6.045 \mathrm{~h}$ as the measured synodic period. The other prominent minima at $3.022 \mathrm{~h}$ is simply the result of folding the lightcurve at half the period, resulting in low fit residuals, which is expected for an asteroid lightcurve with near sinusoidal shape. If the lightcurve had a more asymmetric shape, then the periodogram feature around $3.022 \mathrm{~h}$ would be much less prominent.

Our final fit, using a 5 th order polynomial to refine the period estimate above, is shown in the lower panel of Fig. 4. Prominent minima are seen at approximately 6,8 , and $12 \mathrm{~h}$. The best-fit 

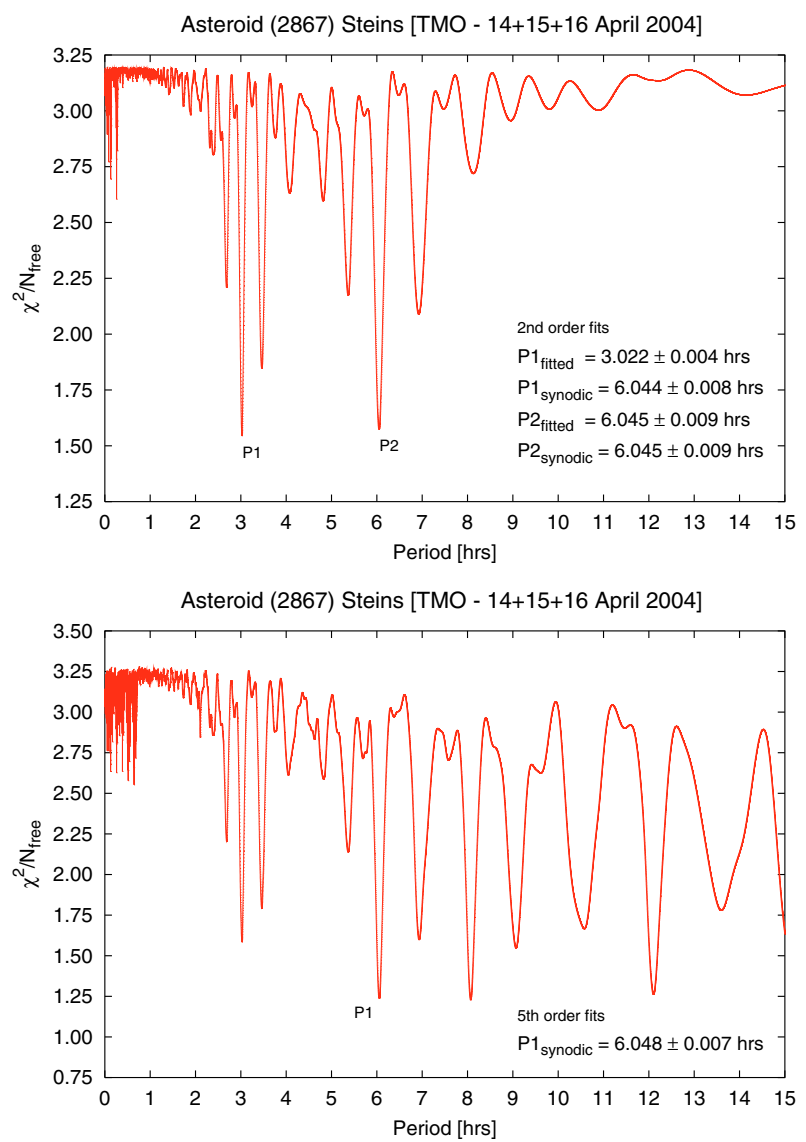

Fig. 4. Upper panel: resulting periodogram from 2nd order Fourier fits to the relative magnitudes. We find a best synodic period of $6.045 \pm$ 0.009 hours. Note the "dual" structure of the periodogram which is expected for an asteroid lightcurve with near sinusoidal shape. Lower panel: periodogram for a 5 th order Fourier series fit to our $R$-filter time series data. These higher order fits were performed in order to improve the accuracy of the period. We find a best-fit synodic rotation period of $6.048 \pm 0.007 \mathrm{~h}$. The feature near $8 \mathrm{~h}$ is not physically realistic as it produces a triple-peaked lightcurve. The other prominent feature near $12 \mathrm{~h}$ is a harmonic of 6.048 .

synodic period is $6.048 \pm 0.007 \mathrm{~h}$. The feature near $8 \mathrm{~h}$ is not physically realistic as it produces a triple peaked lightcurve. The other prominent feature near $12 \mathrm{~h}$ is just a harmonic of 6.048 .

In Fig. 5, we plot the relative magnitudes vs rotational phase. The points are folded to the best-fit synodic period of $6.048 \mathrm{~h}$, and the relative magnitudes for each night have been scaled according to their nightly averages and then centered around zero, so that small geometry changes are accounted for in the folding of the data points. The double-peaked asymmetric lightcurve covering one full rotation is clearly visible each night. Our derived period agrees well with the previous value obtained by Hicks et al. (IAUC 8315), who found a period of $6.06 \pm 0.05 \mathrm{~h}$.

In Fig. 6 we compare our Steins rotational lightcurve parameters with other asteroidal bodies. The overplotted curves are lines of constant bulk density for a simple centrifugal break-up model, and for various density values. For a strengthless prolate ellipsoidal body, the critical rotation period $P_{\text {critical }}-$ beyond which a body will be broken apart by centrifugal forces exceeding self gravity - can be approximated by

$P_{\text {critical }} \approx \frac{3.3}{\sqrt{\rho}} \sqrt{\frac{a}{b}}$

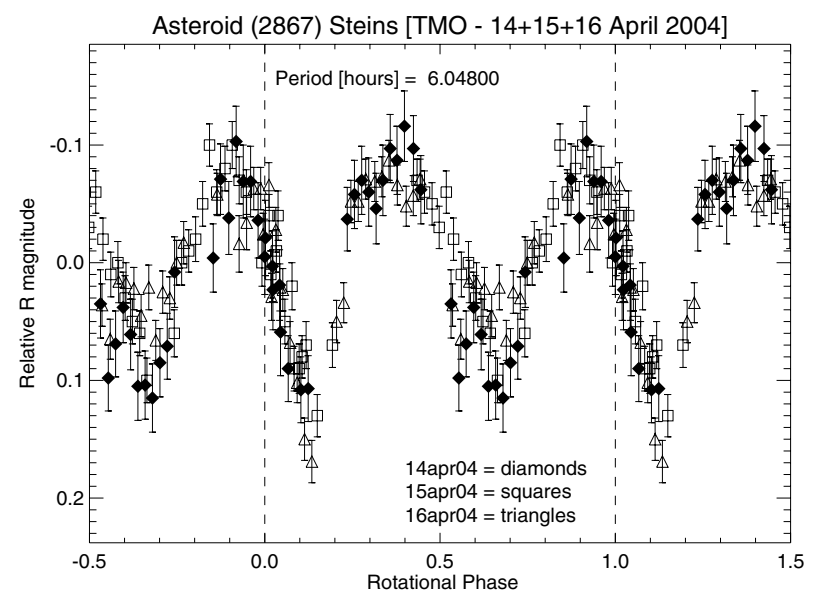

Fig. 5. The $R$-filter relative magnitudes phased to the best-fit synodic period of $6.048 \mathrm{~h}$. Data from different nights are distinguished by the various symbol types. The repeatability from night to night is excellent. The full brightness variation was $0.29 \pm 0.04 \mathrm{mag}$.

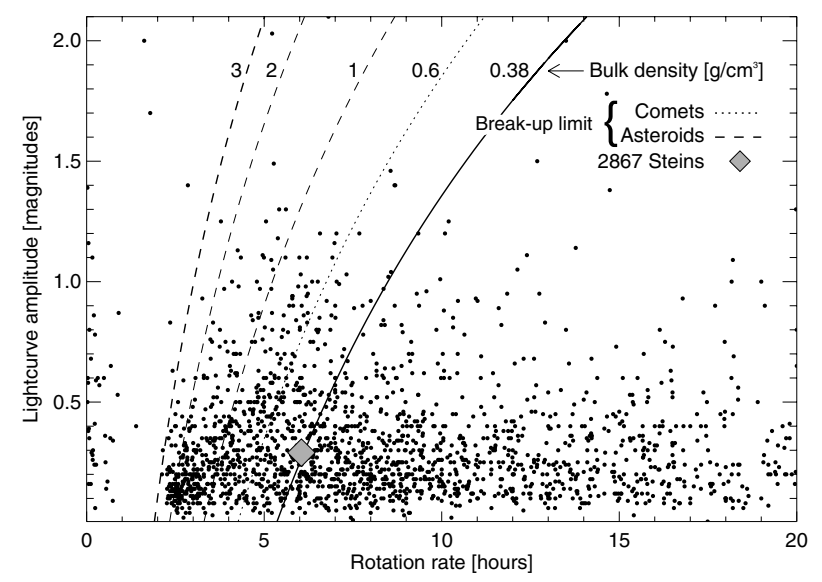

Fig. 6. Comparison of our Steins rotational lightcurve parameters with other asteroidal bodies. The curves are lines of constant bulk density for a simple centrifugal break-up model (see text). The rubble-pile breakup limit is shown for the asteroid population at a density of $3 \mathrm{~g} / \mathrm{cm}^{3}$. The equivalent break-up limit for cometary nuclei at $0.6 \mathrm{~g} / \mathrm{cm}^{3}$ is also marked. The location of Steins on this chart indicates that this asteroid is very typical of asteroidal bodies. From this model we measure a bulk density lower limit for Steins of $0.38 \mathrm{~g} / \mathrm{cm}^{3}$.

where $P_{\text {critical }}$ is in units of hours, $\rho\left[\mathrm{g} / \mathrm{cm}^{3}\right]$ is the bulk density of the body, and $a / b$ is the axial ratio derived from the lightcurve amplitude as described in Sect. 3.1 (Pravec \& Harris 2000). $P_{\text {critical }}$ and $\rho$ are derived directly from the lightcurve, although the latter is only a lower limit as the rotation axis orientation is unknown and the derived axial ratio is the projected value. Also, there is no requirement that the asteroid be rotating at its critical period. Therefore one can only obtain a lower limit to the bulk density from the lightcurve parameters.

The rubble-pile breakup limit is shown for the asteroid population at a density of $3 \mathrm{~g} / \mathrm{cm}^{3}$. The only asteroids that are able to spin faster than the $\sim 2 \mathrm{~h}$ spin rate are the so-called monolithic fast rotators. The equivalent break-up limit for cometary nuclei is also marked at $0.6 \mathrm{~g} / \mathrm{cm}^{3}$ (Lowry \& Weissman 2003). One can see that Steins is very typical in terms of rotation period and elongation. We applied this simple break-up model to Steins to place a bulk density lower limit of $0.38 \mathrm{~g} / \mathrm{cm}^{3}$. Strictly speaking, the full magnitude range is not yet known, which could affect this density lower limit determination. 


\section{Discussion and summary}

We have analyzed results from photometric observations of the Rosetta mission flyby target asteroid 2867 Steins, obtained on April 14-16, 2004 (UT) at the Table Mountain Observatory, California. These data, when combined with future data at different observing geometries, will be critical for developing a detailed 3D shape and orientation model (crucial for Rosetta's science and operations planning), as well as allowing surface properties to be investigated in detail prior to the flyby in September 2008. Our main conclusions are as follows:

1. We measured a mean apparent $R$-filter magnitude of $16.80 \pm$ 0.02 . If we assume a phase-slope parameter $\mathrm{G}$ of 0.15 , as used by Hicks et al. (IAUC 8315), we obtain a mean absolute $R$-filter magnitude of $12.60 \pm 0.02$. This is slightly brighter than the Hicks et al. measurement but consistent at the $2 \sigma$ level. If we assume a typical E-type-asteroid $G$ value of 0.40 (the type assigned to Steins by Barucci et al. 2005) we obtain a mean absolute $R$-filter magnitude of $12.86 \pm 0.02$.

2. The observed rotational lightcurve was asymmetric, and the observed full brightness range was $0.29 \pm 0.04 \mathrm{mag}$. This implies an axial ratio, $a / b$, of $\geq 1.30 \pm 0.04$.

3. We fitted a 5 th order Fourier series to the time-series relative magnitudes and found a best-fit synodic rotation period of $6.048 \pm 0.007 \mathrm{~h}$, in good agreement with other observers. The rotation rate and shape of Steins is very typical for asteroidal bodies. Using a centrifugal break-up model we determined a bulk density lower limit of $0.38 \mathrm{~g} / \mathrm{cm}^{3}$.

4. Assuming a typical S-type albedo of 0.20 (and $G=0.15$ ), our mean apparent $R$-band magnitude implies a mean effective radius of $3.57 \pm 0.03 \mathrm{~km}$ or semi-axes of $3.42 \pm 0.03$ and $4.06 \pm 0.03 \mathrm{~km}$ if the full phase-angle-corrected magnitude range is considered. Assuming a typical E-type albedo of 0.40 (and $G=0.40$ ), we obtain a smaller mean effective radius of $2.24 \pm 0.02 \mathrm{~km}$ or semi-axes of $2.14 \pm 0.02$ and $2.54 \pm 0.02 \mathrm{~km}$.

5. We use the available $R$-filter photometry for a preliminary assessment of the phase-angle variation of the asteroid's brightness. We combined our mean apparent magnitudes with the Hicks et al. data (IAUC 8315) to perform a fit of the photometric phase function in terms of the HG formalism. The best fit phase function parameters and associated $1 \sigma$ uncertainties are $G=0.46_{-0.20}^{+0.32}$, and $H=12.92_{-0.17}^{+0.22}$. Opposition-surge coverage would be useful along with observations at large phase angles in order to refine this measurement.
6. Our measured colour indices are: $(V-R)=0.58 \pm 0.03$, $(R-I)=0.44 \pm 0.03$, and thus $(V-I)=1.03 \pm 0.04$. These values agree well with Hicks et al. (IAUC 8315) but suggest that if Steins is an E-type asteroid as suggested by Barucci et al. (2005), then it is an unusually red E-type object.

Acknowledgements. We thank the referees for their comments on an earlier draft of this paper. This work was performed in part at the Jet Propulsion Laboratory under a contract with NASA and at Queens University, Belfast. This work was funded in part by the NASA Rosetta and Planetary Astronomy Programs. SCL gratefully acknowledges support from the Leverhulme Trust. Table Mountain Observatory is operated under internal funds from JPL's Science and Technology Management Council. IRAF is distributed by the National Optical Astronomy Observatories, which is operated by the Association of Universities for Research in Astronomy, Inc. We acknowledge JPL's Horizons online ephemeris generator for providing the asteriod's position and rate of motion during the observations.

\section{References}

Abe, M., Takagi, Y., Kitazato, K., et al. 2006, Science, 312, 1334

Barucci, M. A., Fulchignoni, M., Fornasier, S., et al. 2005, A\&A, 430, 313

Bowell, E., Hapke, B. Domingue, D., et al. 1989, In Asteroids II (Tucson: Univerisity of Arizona Press), 524

Dandy, C. L., Fitzsimmons, A., \& Collander-Brown, S. J. 2003, Icarus, 163, 363

Fornasier, S., Belskaya, I., Fulchignoni, M., Barucci, M. A., \& Barbieri, C. 2006, A\&A, 449, L9

Fujiwara, A., Kawaguchi, J., Yeomans, D. K., et al. 2006, Science, 312, 1330

Giorgini, J. D., \& Yeomans, D. K. 1999, NASA Tech Briefs, NPO-20416, 48

Harris, A. W., Young, J. W., Bowell, E., et al. 1989, Icarus, 77, 171

Hicks, M. D., Bauer, J. M., \& Tokunaga, A. T. 2004, IAU Circ, 8315

Kaasalainen, M., Kwiatowski, T., Abe, M., et al. 2003, A\&A, 405, L29

Landolt, A. U. 1992, AJ, 104, 340

Lamy, P. L., Jorda, L., Fornasier, S., et al. 2006, BAAS, 38, \#59.09

Lederer, S. M., Domingue, D. L., Vilas, F., et al. 2005, Icarus, 173, 153

Lowry, S. C., \& Weissman, P. R. 2003, Icarus, 164, 492

Pravec, P., \& Harris, A. W. 2000, Icarus, 148, 12

Russell, H. N. 1916, ApJ, 43, 173

Tedesco, E. F. 1989, In Asteroids II (Tucson: University of Arizona Press), 1090

Tholen, D. J. 1984, Asteroid taxonomy from the cluster analysis of photometry, Ph.D. thesis, Univ. of Arizona

Tody, D. 1986, in Proc. SPIE Instrumentation in Astronomy VI, 627, 733

Tody, D. 1993, in Astronomical Data Analysis Software and System II, ASP Conf. Ser., 52, 173

Zappalà, V., Cellino, A., Barucci, A. M., Fulchignoni, M., \& Lupishko, D. F. 1990, A\&A, 231, 548

Zellner, B., Tholen, D. J., \& Tedesco, E. F. 1985, Icarus, 61, 355 